\title{
Treatment-required accidental ingestion and risk factors among nursery children with wheat allergy
}

\author{
Noriyuki Yanagida $^{1}$, Motohiro Ebisawa ${ }^{1}$, Toshio Katsunuma ${ }^{2}$, and Jyoji Yoshizawa ${ }^{3}$ \\ ${ }^{1}$ Sagamihara National Hospital \\ ${ }^{2}$ The Jikei University Daisan Hospital \\ ${ }^{3}$ The Jikei University School of Medicine
}

January 25, 2021

\section{Treatment-required accidental ingestion and risk factors among nursery children with wheat allergy}

\section{Conflicts of interest}

M. Ebisawa serves in the clinical medical advisory board of DBV Technologies. Motohiro Ebisawa have received speaker honoraria from Mylan EPD. All other authors declare no conflicts of interest.

\section{Financial support}

This study was supported by funds from the Ministry of Health, Labour and Welfare of Japan.

\section{To the Editor:}

A nationwide questionnaire-based survey conducted throughout nursery schools in Japan revealed that 7.6\% children have accidental ingestion within one year. ${ }^{1}$ Younger age, male sex, and wheat allergy are risk factors for accidental ingestion. ${ }^{2}$ However, the frequency of accidental ingestion of each allergen and risk factors for treatment-required moderate-to-severe accidental ingestion remain unknown in nursery schools. This study examined the prevalence of accidental ingestion of four common allergens and risk factors for treatmentrequired accidental ingestion in nursery schools in Japan. ${ }^{2}$

Data were analyzed using a large dataset from a previous nationwide questionnaire survey, entitled "Survey on allergic disease status of children admitted to nursery schools and allergy measures at nursery schools." Webbased survey forms containing instructions were dispatched to all childcare facilities in Japan through post (Table S1) ${ }_{1}^{1}$ covering two-thirds of preschool children who attended nursery schools in Japan (Supplementary Information). The survey had questions about the presence of accidental ingestion between April 2015 and February 2016 and details about the accidental ingestion. We enrolled children with egg, milk, wheat, or peanut allergy (four common allergens) and excluded children with allergy to multiple foods or to foods other than egg, milk, wheat, or peanut. We also excluded patients with missing data.

This study was approved by the ethics committee of Jikei University. Informed consent from the guardians of the participating children was not required because no personal data that identified the participants were obtained. Detailed methods of the survey administered to nursery schools and the regulations in Japan are listed in Supplementary Information.

Food allergy was defined as doctor-diagnosed IgE-mediated food allergy ${ }^{3}$ with written evidence submitted to the nursery school. If children experienced multiple episodes of allergic symptoms due to accidental ingestion, the most severe episode was investigated. Accidental ingestion was defined as unintended accidental allergen 
intake leading to allergic symptoms. Treatment-required accidental ingestion was defined as accidental ingestion that required treatment at the clinic or hospital.

Data are expressed as n (\%) or arithmetic mean (standard deviations). Fisher's exact tests, with Bonferroni correction and Mann-Whitney U tests, were used, and $p<0.05$ was considered statistically significant. Logistic regression was used to perform multivariate analysis. SPSS Statistics for Windows, version 25.0 (IBM Corp., Armonk, NY, USA), was used for statistical analyses.

In total, 1,390,481 children were enrolled in the survey (Figure S1). Among 56,121 children with food allergy, 10,315 were excluded owing to missing data. We further excluded 17,794 children with allergy to multiple foods and 5,251 children with allergy to foods other than egg, milk, peanut, or wheat. Finally, 22,761 children with egg, milk, wheat, or peanut allergy met the inclusion criteria. Mean age of the children was $2.0 \pm 1.5$ years (median: 2.0 years) (Table 1 ). Egg allergy was most common, followed by allergy to milk, peanuts, and wheat (Table S1). Overall, $1383(6.1 \%)$ children experienced accidental ingestion and presented with symptoms during the last 1 year, $78(5.6 \%)$ of whom required treatment at the clinic or hospital and $12(0.9 \%)$ were hospitalized. Only 1 child $(0.01 \%)$ used an adrenaline auto-injector in their nursery school. There were no fatal cases requiring treatment in the intensive care unit or resuscitation. Misdistribution of meals was the most common reason for accidental ingestion (31.3\%) and eating other children's meal was the second common reason $(11.9 \%)$.

Accidental ingestion correlated with male sex, younger age, and history of anaphylaxis to causative food (Table 1). The incidence of accidental ingestion was highest (10.6\%) in wheat allergic children and significantly higher than that in egg $(5.9 \%)(p=0.001)$ and peanut $(5.3 \%)$ allergy $(p=0.011)$, even after Bonferroni correction (Figure 1). Significant differences were not seen in milk $(6.8 \%)$ allergy after Bonferroni correction $(p$ $=0.07)$. Similarly, among children with accidental ingestion, the incidence of treatment-required accidental ingestion was most common in wheat allergy $(31.4 \%)$, followed by peanut $(18.8 \%)(p=0.49)$, milk $(10.7 \%)$ $(p=0.019)$, and egg $(8.6 \%)(p<0.001)$ allergy (Figure 1$)$.

Risk factors for treatment-required accidental ingestion were assessed among children with accidental ingestion during the last 1 year (Table 2). Multivariate analysis revealed the factors significantly associated with treatment-required accidental ingestion for adjusted odds ratio (aOR) adjusted for parameters listed in Table 1. History of anaphylaxis to causative foods was a significant risk factor in egg (aOR: 3.069), milk (aOR: 5.325), peanut (aOR: 30.286), and wheat (aOR: 11.721) allergy. Younger age was a significant risk factor in egg (per one-year increase; aOR: 0.758) and milk (per one-year increase; aOR: 0.668) allergy.

Among wheat allergic children with a history of anaphylaxis to wheat $(\mathrm{n}=69), 16(23.2 \%)$ experienced accidental ingestion (Figure S2). Nine $(36.3 \%$ ) of the 16 patients had treatment-required symptoms.

Our study is the first to confirm the potential risk of accidental ingestion of wheat in the general setting of nursery schools based on a nationwide survey.

Although our previous results indicated that wheat allergy was a major risk factor for accidental ingestion among nursery school children in Japan ${ }^{2}$, the study design could not clarify the incidence of accidental ingestion among different food allergens in participants with multiple food allergy. In this study, we compared the frequency and severity of symptoms due to accidental ingestion of each allergen. Wheat allergic children had accidental ingestion more frequently than children allergic to foods other than wheat. Wheat is one of the most lethal food allergens in the Japanese population, often inducing anaphylaxis ${ }^{4}$. Reactions that require treatment are more common in oral food challenges (OFCs) to wheat than in OFCs to other foods. ${ }^{5,6}$. Our results are similar to the results of previous studies. The prevalence of wheat allergy differs between countries, ${ }^{7}$ and wheat allergy is more common in Asian countries than in European countries and the United States. ${ }^{8,9}$ Wheat is the third most common allergen and the primary cause of anaphylaxis in Japan. ${ }^{3}$ Therefore, we should consider common allergens that cause anaphylaxis in preschool children according to the country.

A history of anaphylaxis to causative food was a risk factor for treatment-required accidental ingestion. A history of anaphylaxis is a risk factor for severe reaction during OFC among school-aged high-risk patients. ${ }^{4}$ 
In a study of anaphylaxis recurrence, one-fifth of children with a history of food anaphylaxis experienced at least one anaphylaxis recurrence at a mean interval of 12 months. ${ }^{10}$ We confirmed similar results in younger and generalized populations. Indeed, one-fourth of the children with a history of anaphylaxis had accidental ingestion within 1 year, and one-third of them were treated in the hospital or clinic for accidental ingestion (Figure S2). Therefore, we should consider the causative food type and a history of anaphylaxis when children enter nursery school to prevent accidental ingestion.

Our study had several limitations. The study is a questionnaire-based survey and therefore has the potential for bias. We excluded many patients with multiple food allergens known as risk factors for recurrent anaphylaxis, ${ }^{10}$ which may indicate selection bias. This survey did not consider complications including bronchial asthma and allergic rhinitis, which may affect the severity of accidental ingestion.

In conclusion, we should recognize and understand the difference in the incidence of accidental ingestion induced by each food allergen and risk factors for treatment-required accidental ingestion, including a history of anaphylaxis in nursery school children. To generalize these results, further prospective studies on accidental ingestion in other populations will be needed.

Keywords : Accidental exposure, Anaphylaxis, Food allergy, Ingestion, Nursery schools, Pediatric, Risk factors

Noriyuki Yanagida, $\mathrm{MD}^{\mathrm{a}, \mathrm{b}}$, Motohiro Ebisawa, $\mathrm{MD}, \mathrm{PhD}^{\mathrm{b}}$, Toshio Katsunuma, $\mathrm{MD}, \mathrm{PhD}^{\mathrm{c}}$, Jyoji Yoshizawa, $\mathrm{MD}, \mathrm{PhD}^{\mathrm{d}}$

${ }^{a}$ Department of Pediatrics, National Hospital Organization, Sagamihara National Hospital, Kanagawa, Japan

bJapan Clinical Research Center for Allergy and Rheumatology, National Hospital Organization, Sagamihara National Hospital, Kanagawa, Japan

${ }^{\mathrm{c}}$ Department of Pediatrics, The Jikei University Daisan Hospital, Tokyo, Japan

${ }^{\mathrm{d}}$ Division of Pediatric Surgery, Department of Surgery, The Jikei University

School of Medicine, Tokyo, Japan

Acknowledgements : We are particularly grateful to the staff members who participated in the survey and data collection. Assistance in English language editing was provided by Editage, Cactus Communications.

\section{References}

1. Yanagida N, Ebisawa M, Katsunuma T, Yoshizawa J. Ministry of Health "2015 Penultimate Year Children and Survey and Research Project Promoting the Parenting Support" Actual conditions report on the results of the investigation on allergy control in day-care centers admissions children of allergic diseases situation and day-care centers]. Arerugi 2018;67:202-210.

2. Yanagida N, Ebisawa M, Katsunuma T, Yoshizawa J. Accidental ingestion of food allergens: A nationwide survey of Japanese nursery schools. Pediatr Allergy Immunol 2019;30:773-776.

3. Ebisawa M, Ito K, Fujisawa T, Committee for Japanese Pediatric Guideline for Food Allergy TJSoPA, Clinical Immunology TJSoA. Japanese guidelines for food allergy 2017. Allergol Int 2017;66:248-264.

4. Akiyama H, Imai T, Ebisawa M. Japan food allergen labeling regulation - history and evaluation. Adv Food Nutr Res 2011;62:139-171.

5. Yanagida N, Sato S, Asaumi T, Ogura K, Ebisawa M. Risk factors for severe reactions during double-blind placebo-controlled food challenges. Int Arch Allergy Immunol 2017;172(3):173-182.

6. Cianferoni A, Khullar K, Saltzman R, et al. Oral food challenge to wheat: a near-fatal anaphylaxis and review of 93 food challenges in children. World Allergy Organ J 2013;6(1):14. 
7. Siripipattanamongkol N, Vichyanond P, Jirapongsananuruk O, Veskitkul J, Visitsunthorn N, Pacharn P. Age of resolution from IgE-mediated wheat allergy. Asian Pac J Allergy Immunol 2017;35(2):113-117.

8. Ricci G, Andreozzi L, Cipriani F, Giannetti A, Gallucci M, Caffarelli C. Wheat allergy in children: A comprehensive update. Medicina (Kaunas) 2019;55(7):400.

9. Cianferoni A. Wheat allergy: diagnosis and management. J Asthma Allergy 2016;9:13-25.

10. Pouessel DG. Recurrent food anaphylaxis and risk factors in children. Pediatr Allergy Immunol 2020. doi: 10.1111/pai.13429. Epub ahead of print.

Table 1. Characteristics of children with food allergy

\begin{tabular}{lllll}
\hline & Total $(\mathrm{n}=22,761)$ & $\begin{array}{l}\text { Accidental ingestion } \\
(\mathrm{n}=1,383)\end{array}$ & $\begin{array}{l}\text { No exposure or no } \\
\text { symptoms due to } \\
\text { accidental ingestion } \\
(\mathrm{n}=21,378)\end{array}$ & $p$-value \\
& $13755(60.4)$ & $877(63.4)$ & $\begin{array}{l}12878(60.2) \\
2.0(1.5)\end{array}$ & 0.020 \\
$\begin{array}{l}\text { Sex (male) }(\mathrm{n}, \%) \\
\begin{array}{l}\text { Age (years) } \\
\text { (mean, SD) }\end{array}\end{array}$ & $2.0(1.5)$ & $1.9(1.5)$ & $1479(6.9)$ & $<0.001$ \\
$\begin{array}{l}\text { History of } \\
\text { anaphylaxis to } \\
\text { causative food (n, }\end{array}$ & $1701(7.5)$ & $222(16.1)$ & & $<0.001$ \\
$\%)$ & & & \\
\hline
\end{tabular}

SD, standard deviation

Accidental ingestion was defined as unintended accidental exposure to the allergen leading to allergic symptoms.

At least $83.3 \%$ of the children submitted documents with details about their food allergies and related instructions, written by their doctors, to their nursery school.

Table 2. Risk factors for treatment-required accidental ingestion among children with accidental ingestion during the last 1 year

\begin{tabular}{|c|c|c|c|c|}
\hline & $\begin{array}{l}\text { Adjusted OR* } \\
(95 \% \text { CI })\end{array}$ & $\begin{array}{l}\text { Adjusted OR* } \\
(95 \% \text { CI })\end{array}$ & $\begin{array}{l}\text { Adjusted OR* } \\
(95 \% \text { CI })\end{array}$ & $\begin{array}{l}\text { Adjusted OR* } \\
(95 \% \mathrm{CI})\end{array}$ \\
\hline & $\operatorname{Egg}(n=1118)$ & Milk $(\mathrm{n}=198)$ & Peanut $(\mathrm{n}=32)$ & Wheat $(\mathrm{n}=35)$ \\
\hline \multirow[t]{2}{*}{ Sex (male) } & $0.764(0.411-1.420)$ & $0.521(0.189-1.432)$ & $2.100(0.114-38.751)$ & $0.807(0.122-5.315)$ \\
\hline & $p=0.394$ & $p=0.206$ & $p=0.618$ & $p=0.823$ \\
\hline \multirow[t]{2}{*}{ Age (years) } & $0.758(0.593-0.968)$ & $0.668(0.450-0.994)$ & $1.400(0.495-3.955)$ & $0.784(0.472-1.301)$ \\
\hline & $p=0.027$ & $p=0.046$ & $p=0.526$ & $p=0.346$ \\
\hline History of & $3.069(1.579-5.964)$ & $5.325(1.936-14.641)$ & 30.286 & 11.721 \\
\hline $\begin{array}{l}\text { anaphylaxis to } \\
\text { causative food }\end{array}$ & $p=0.001$ & $p=0.001$ & $\begin{array}{l}(1.982-462.843) p= \\
0.014\end{array}$ & $\begin{array}{l}(1.847-74.394) p= \\
0.009\end{array}$ \\
\hline
\end{tabular}

OR, odds ratio; CI, confidence interval.

*OR was adjusted using the significant variables shown in Table 1: sex, age, and history of anaphylaxis to causative foods.

Odds ratio of "Age" per one-year increase

Risk factors for treatment-required accidental ingestion were examined among children with accidental in- 
gestion between April 2015 and February 2016. All children with treatment-required accidental ingestion received treatment at a clinic or hospital.

\section{Figure legend}

Figure 1: Proportion of children who developed symptoms of food allergy in their nursery school during the last 1 year.

This figure shows the proportion of children who developed symptoms of food allergies in their nursery school between April 2015 and February 2016.

Among children with accidental ingestion, the incidence of accidental ingestion is most common in children with allergy to wheat $(31.4 \%)$, followed by children with allergy to peanut $(18.8 \%)(\mathrm{p}=0.49)$, allergy to milk $(10.7 \%)(\mathrm{p}=0.019)$, and allergy to egg $(8.6 \%)(\mathrm{p}<0.001)$.

All children with treatment-required accidental ingestion received treatment at a clinic or hospital.

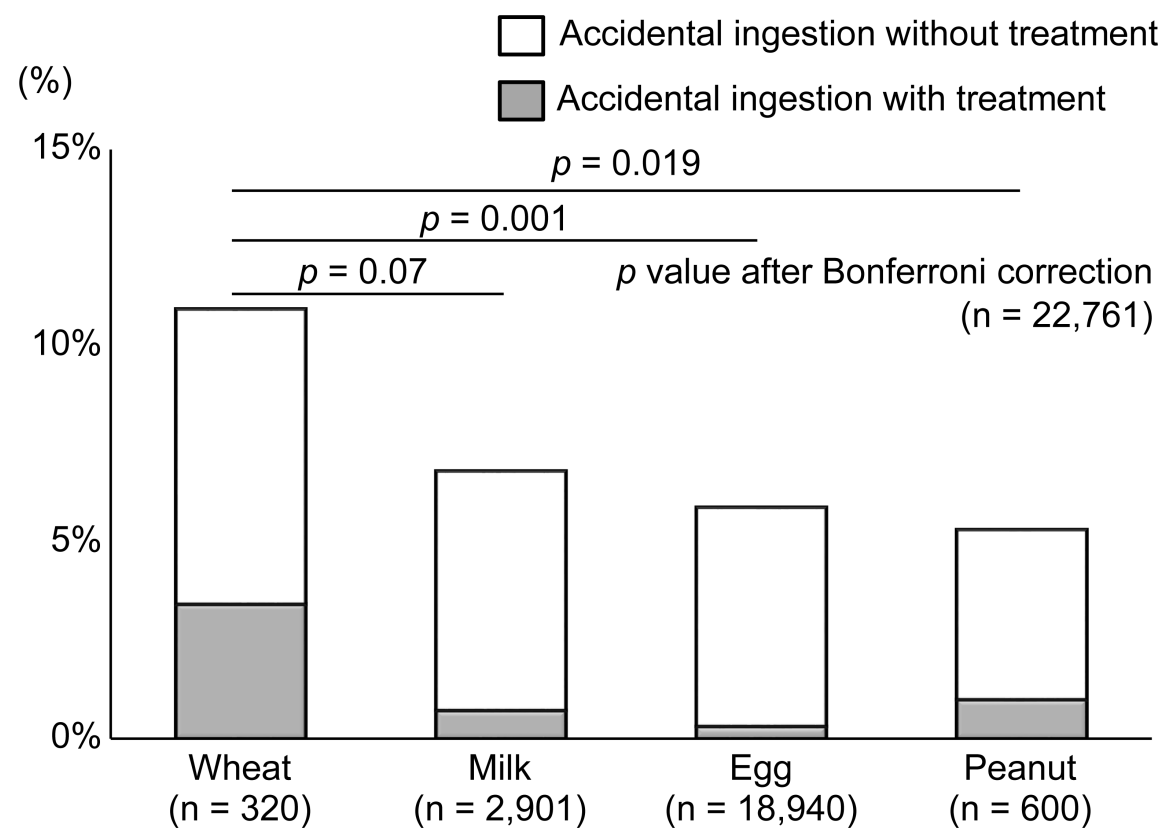

